Reidel, 1978), and the meeting on photoelectrochemistry to be held in Cambridge this August will give an up-to-date review of the rapid progress in this new and exciting field. It looks as if 'wet' semiconductor devices are here to stay.

\section{Accelerator dating techniques}

from Terry S. Mast

A DRAMATICALLY more sensitive method for measuring low concentrations of radioisotopes has generated a great deal of excitement in the field of radioisotope dating, and stimulated a remarkably large development effort in a short amount of time. Twenty months of development, which is expected to have substantial impact on many fields of research, including archeology, geology, oceanography, and astrophysics, were reviewed at a recent conference*.

The basic idea of the technique is really a synthesis of two old ideas. The first is the idea of counting directly the radioisotope atoms in a sample rather than waiting for them to decay (Muller Science 196, 489; 1977). The potential gain in sensitivity is apparent when one realises that in a sample being dated with ${ }^{14} \mathrm{C}$ which has a typical decay rate of 1 decay per minute there are about $10^{9}$ atoms of ${ }^{14} \mathrm{C}$. Attempts to count these directly with mass spectrometers have been unsuccessful because the very low concentration of ${ }^{14} \mathrm{C}\left(10^{-12}\right.$ to $\left.10^{-34}\right)$ is inevitably masked by a much larger contamination of ${ }^{14} \mathrm{~N}$. The second idea, which solves the problem of the ${ }^{14} \mathrm{~N}$, is to use an accelerator as a mass spectrometer. Ionising and accelerating the sample atoms to a high energy (40-50 MeV) allows the separation of the ${ }^{14} \mathrm{~N}$ and identification of the ${ }^{14} \mathrm{C}$ using the particle identification techniques of nuclear physics. The standard technique of counting the beta decays of the ${ }^{14} \mathrm{C}$ typically requires $1-10 \mathrm{~g}$ carbon and takes from $2-24 \mathrm{~h}$ to count. The new technique enables measurements to be made in $1 \mathrm{~h}$ or less with less than 5 to $10 \mathrm{mg}$ carbon.

Physicists described designs for accelerator-detector systems for isotope dating and the results of the development tests being made. Achaeologists, geochemists and cosmochemists outlined new studies which can be made with the higher sensitivity, and how measurement reliability for existing studies could be proved.

Both cyclotrons and tandem Van de Graaff accelerators have been used

*Held at the University of Rochester, New York, from 20-21 April. now to detect several radioisotopes at natural concentrations (Nelson et al. Science 198, 507; 1977). The emphasis so far has been on ${ }^{14} \mathrm{C}$ (half life $=5730 \mathrm{yr}$; used extensively in archaeology) and on ${ }^{10} \mathrm{Be}$ (half life = $1.5 \times 10^{6} \mathrm{yr}$; used for geochronology) There are at present six research groups making measurements and many others interested in starting programmes.

Groups at the University of California Lawrence Berkeley Laboratory and the Laboratory Rene Bernas, Orsay, have used cyclotrons. The samples are ionised in a Penning lon Gauge source and accelerated. The cyclotron resonance condition sharply defines the charge: mass ratio accepted, and only ions with almost identical masses are accelerated-for example, ${ }^{14} \mathrm{C}$ and ${ }^{14} \mathrm{~N}$ or ${ }^{10} \mathrm{Be}$ and ${ }^{10} \mathrm{~B}$. The radioisotope and stable isotope are then cleanly separated by their different ranges in an absorber. As the contaminating stable isotope is typically $10^{10}$ times more abundant in the beam than the radioisotope, the separation must be and is extremely efficient.

Tandem accelerators are being used by a large collaboration between the University of Rochester, the University of Toronto, and the General Ionex Corporation (Massachusetts): by a group at Chalk River, Canada; by a collaboration between the Simon Fraser and McMaster's Universities; and by a group at Oxford University. The beam for the tandem work is produced by a caesium ion sputter source, which for ${ }^{14} \mathrm{C}$ dating sputters $\mathrm{C}^{-}$from a solid carbon sample. The separation of the ${ }^{14} \mathrm{~N}$ comes from the fact that nitrogen only forms metastable negative ions which decay before passing through the tandem accelerator. A number of other contaminating isotopes, which are accelerated through the tandem, as well as the residual ${ }^{14} \mathrm{~N}$, are then cleanly separated from the ${ }^{14} \mathrm{C}$ by magnetic analysis and an ion identification detector telescope.

All groups have successfully solved the problems of detecting the radioisotopes cleanly separated from contaminating stable isotopes and with a sensitivity sufficient to measure the very low concentrations found in nature. The future development work will focus on accurate normalisation schemes, proof of reliable accuracy, and finally improvement of precision.

The main benefit of the improved sensitivity will be the ability to determine the isotope concentration with only a few milligrams of sample. This will allow the dating of many objects

Terry S. Mast is at the University of California Lawrence Berkeley Laboratory and Space Sciences Laboratory. which are too small to date with decay counting. Perhaps more important will be the ability to select components of a sample (such as particular proteins) which are specific to the object being dated, thus reliably removing other sources of carbon that might have leached into the object. Finally, the ability to measure several samples from the same artefact will enable checks on contamination removal procedures to be made.

The development work has so far been done on a few radioisotopes${ }^{14} \mathrm{C}$, ${ }^{10} \mathrm{Be}$ and ${ }^{36} \mathrm{Cl}$. Participants at the conference emphasised that a large number of other radioisotopes have been used in geochronology and cosmochronology and that the new method will be useful in extending that research as well.

\section{Crystal defects and melting}

from Robert W. Cahn

OF all the common properties of crystals, melting has a claim to be considered the most mysterious. A1though there is no dearth of hypotheses, we do not know with certainty what structural features different crystal species have in common at the instant of melting.

In recent years, two venerable but mutually incompatible theories have been taken out and dusted, and their current champions each claim to have established strong evidence in their respective favour. The first is the dislocation theory of melting, first propounded by J. K. Mackenzie and N. F. Mott in 1952. The basic idea is that the energy of a dislocation array is a function both of dislocation concentration and of temperature (if dislocations are close together they partly relieve each other's stress fields), and if the temperature is high enough then a sudden catastrophic increase in dislocation density is predicted, to the point at which all crystallinity is lost. The early work is summarised by A. R. Ubbelohde (Melting and Crystal Structure, Oxford University Press, 1965).

R. J. M. Cotterill, W. D. Kristensen and E. J. Jensen have carried out extensive computer simulations, in two dimensions, which have shown that as kinetic energy is progressively injected, increasing populations of dislocation loops are created, grow and collapse again. When the instantaneous population grows large enough, the expanding loops "literally cut the crystal

Robert W. Cahn is Professor of Materials Science at the University of Sussex. 